\title{
CUESTIONES TEÓRICAS PLANTEADAS POR LA INTEGRACIÓN ECONÓMICA REGIONAL
}

\author{
Havelock Brewster y Clive Thomas * \\ University of the West Indies
}

\section{INTRODUCCIÓN}

En el campo de las relaciones económicas internacionales, la mayor parte de los temas se encuentran hoy día subordinados a la búsqueda de asociaciones o agrupaciones económicas regionales entre áreas más o menos contiguas. El "éxito" de la Comunidad Económica Europea ha inspirado en gran medida este tipo de orientación que ha encontrado a su vez apoyo casi unánime en diversos países del mundo. La idea de la integración ha sido respaldada también ampliamente entre los economistas. Al examinar la literatura sobre el tema, nos ha extrañado encontrar muy escasa fundamentación teórica de esta orientación tan general hacia la "cooperación" económica. La teoría de la integración existente constituye en gran medida un desarrollo de los postulados neoclásicos de Viner concernientes a la "desviación del comercio" y a la "creación del comercio". Se supone que comprende "esa rama de la teoría arancelaria que trata de los efectos de los cambios geográficamente discriminatorios en las barreras arancelarias"."

Ese enfoque limitado no plantea problemas serios a las sociedades europeas económicamente "maduras" de la Comunidad Económica Europea. Sin embargo, el intento de aplicar en forma indiscriminada este marco teórico y este cuerpo de ideas al análisis de los problemas de integración económica entre economías eccnómicamente "inmaduras" debe ser motivo de honda preocupación, puesto que ciertamente hemos encontrado que aun cuando se hacen intentos de tratar el problema dentro del contexto de los países de menor desarrollo, persiste el dominio de los métodos silogísticos de la teoría tradicional. Únicamente se oscurecen mediante la aplicación aforística de los términos "dinámica" y "estática" tomados de la física. Estas aplicaciones adquieren principalmente la forma de reinterpretaciones de la naturaleza de la desviación o la creación del comercio y de proposiciones

* Este documento fue presentado originalmente en la Conferencia sobre Relaciones Internacionales del Hemisferio Occidental y la Zona del Caribe, auspiciada por El Colegio de México, la Universidad de los Andes, la Universidad de Miami y la Universidad de las Indias Occidentales y que se llevó a cabo en esta última, en Kingston, Jamaica, en mayo de 1968. El trabajo presentado deriva de nuestro libro The Dynamics of West Indian Economic Integration, University of the West Indies, 1967. También es reflejo de un trabajo teórico de mayor amplitud que estamos preparando. La traducción al español es de Clara Jusidman.

1 R. G. Lipsey, "The Theory of Customs Unions: A General Survey", Economic Journal, septiembre de 1960. 
hipotéticas sobre los efectos de la inversión, la tecnología y la relación de precios del intercambio.

Debido a limitaciones de espacio no pretendemos tratar en forma amplia todas las cuestiones teóricas pertinentes. Por lo tanto, hemos aislado una tipología particular de economías económicamente "inmaduras", a saber, economías pequeñas, estructuralmente dependientes (de las cuales son buenos ejemplos la Zona del Caribe, los países de América Central, etc.). Elegimos esta tipología de economías por creer que comprendemos mejor que otros las leyes de funcionamiento que las caracterizan. También hemos aislado cuatro temas para nuestros comentarios, a saber: el concepto de integración económica; el concepto de transformación estructural en economías abiertas de tamaño reducido y su relación con la integración económica; la transformación estructural y los ilmites al crecimiento $\mathrm{y}$, por último, algunas observaciones sobre el papel que desempeña la agricultura en la síntesis de una teoría dinámica de ia integración económica. De la lista de tópicos que nos proponemos tratar puede observarse que para nosotros la teoría de la integración económica en estas economías está íntimamente ligada a la teoría del proceso de desarrollo.

\section{I}

En vista del apoyo casi universal dado a las asociaciones y agrupaciones económicas y sociales de una región dada. sino que debe exintegración económica no haya sido objeto de análisis sistemático mayor. Ciertamente se han llevado a cabo algunos esfuerzos en este sentido, pero, en gran medida, subsisten mucha ambigüedad y mal entendimiento que han obstruido el desarrollo satisfactorio de la teoría de la integración económica. Si bien no pretendemos esclarecer en esta breve sección todas o gran parte de estas dificultades, quisiéramos iniciar un examen preliminar del concepto de integración económica tal como lo vemos.

En la discusión económica contemporánea de este concepto mucha de la ambigüedad existente proviene de que no se investigan desde el principio las cualidades que son inherentes a la idea de integración. En la literatura económica el concepto más comúnmente utilizado se ha limitado en gran medida a las estructuras legales formales de asociación internacional, las cuales pueden variar ampliamente como en efecto ocurre; en cada situación los economistas consideran posible evaluar en forma más o menos correcta los efectos netos positivos o negativos de la integración.

La interpretación que damos al concepto de integración económica es fundamentalmente orgánica. Sus métodos y patrones de desarrollo se realizan a través de la difusión de atributos de fuerza y debilidad a todas las partes integrantes de un sistema regional. Es necesario que esto suceda en tal forma que el balance compensatorio de estos atributos destruya su localización y confiera a cada uno de sus componentes un potencial mayor al que tenían en la situación anterior a la integración. Como tal, implica no sólo la integración de la estructura de la demanda sino también la integración de la utilización 
de los recursos. ${ }^{2}$ De esto se deriva, como debe suceder en cualquier teoría orgánica, que el potencial del sistema integrado debe ser superior al que resulta de la agregación de los componentes individuales, no integrados. Esto significa también que los criterios para valorar los logros de las áreas integradas deben estar relacionados con el área total así como con cada uno de sus componentes.

En este sentido, la integración económica, para que tenga algún significado real, debe ser una idea positiva en relación con cualquiera que sea el potencial que se va a maximizar. Posteriormente aclararemos nuestra propia elección de potencial, derivada de una estrategia particular de desarrollo. Pero en esta etapa es claro que aun cuando la integración no pueda ser concebida negativamente, el potencial a maximizar puede estar sujeto a diferentes juicios de valor. Este enfoque conceptual ha adquirido mayor o menor reconocimiento en la literatura sobre integración social y racial. ¿Por qué no se ha aclarado esto en la literatura sobre integración económica?

Una posible respuesta es la noción mal enfocada de la mayor "objetividad" de nuestra disciplina en comparación con otras disciplinas en las ciencias sociales. ${ }^{3}$ Sin embargo, la integración económica, al igual que otras formas de integración, no puede ser definida simplemente como un mecanismo útil para unir entidades desarticuladas, ya sea por la eliminación de formas de discriminación o a través de la cooperación en la eliminación de conflictos entre metas. Por integración económica uno debe referirse a las consecuencias de cualquiera de los llamados "mecanismos integradores" más que a los mecanismos en sí mismos. Estas consecuencias pueden tener distintos grados positivos, y pueden ser promovidas u obstaculizadas por las diversas formas de asociación. Sin embargo, si el efecto es negativo en términos de los criterios del conjunto y de las partes integrantes, tenemos entonces una situación o proceso de desintegración, cualesquiera que sean las formas de unión en el sistema.

En esta etapa se sugieren dos ejemplos útiles. El primero es el Imperio británico, que en sus días de apogeo acusó diversas formas de asociación que de acuerdo con la interpretación común serían consideradas como integración económica. Por ejemplo, existía movilidad más o menos libre del trabajo y el capital. Había bastante congruencia en cuanto a regulación arancelaria, convenios bancarios y monetarios, política de reservas de divisas, legislación comercial, etc. Existía, además, un gobierno virtualmente unitario. No obstante, muchos de los economistas que prefirieran adoptar una posición menos "extremista" que la nuestra, sostendrían que el desmembramiento de este imperio fue, y continúa siendo, una condición sine qua non para la integración exitosa de muchos países del Tercer Mundo. Más aún, estarían también de acuerdo en que a pesar de los cientos de años de existencia de estos verdaderos lazos financieros y comerciales no se logró nada parecido a una integración económica mutuamente aceptable.

2 Posteriormente se aclararán sus implicaciones económicas precisas.

"No sólo la noción de mayor "objetividad" está mal enfocada sino que la interpretación científica misma de la "objetividad" sugiere algunas dudas metodológicas. 
El otro ejemplo está dado en las Jimitaciones de las "economías de planta subsidiaria" que proliferan en el Tercer Mundo. Tomemos el Caribe como ejemplo. Aquí nos encontramos con una situación en la cual unas cuantas grandes compañías internacionales controlan to dos los "puntos de mando" de estas diversas economías, a saber, la bauxita, el azúcar, el banano y las actividades financieras. Como resultado de esta estructura de empresas, existe un llamado "potencial para la integración" de los recursos de la región a través de las casas matrices de las mismas. Pero dicha integración está sometida a las consideraciones globales de estas compañías y a su búsqueda de ganancias en el total de sus operaciones. Huelga agregar que estas operaciones están integradas verticalmente a las actividades manufactureras finales localizadas en las zonas metropolitanas. En consecuencia, este sistema de "economías de planta subsidiaria" puede ser dis. funcional, y en la región del Caribe lo ha sido realmente, desde el punto de vista de las aspiraciones regionales.

Una interpretación orgánica del concepto de integración económica como la que sugerimos implica necesariamente también que la teoría de la integración económica debe ser un elemento integral de una teoría sobre la transformación económica y social y no un simple agregado de la microeconomía en la teoría estática de la localización. Más aún, significa que el interés no debe limitarse a las transformaciones económicas regionales, asombra en verdad que el concepto de tenderse a las de sus partes constitutivas. De este modo, si se hace un examen cuidadoso de varias economías del mundo es posible observar que en muchos casos el estado desintegrado de un país en particular puede constituirse en un obstáculo mayor a la integración económica de una región que los problemas relativos a la soberanía, etc., entre los países.

Es preciso tener en cuenta que la interpretación aquí propuesta se aparta en forma significativa de la teoría tradicional de la integración económica. Balassa, uno de los que han contribuido en forma más sobresaliente a este campo de la investigación, sostiene: "Por lo tanto, la integración económica nacional hace surgir la discriminación entre unidades económicas de países independientes y de este modo contribuye a la desintegración en la escena internacional... Al excluir del concepto la integración nacional, podemos definir la integración económica como un proceso y un estado de cosas. La integración económica como proceso representa en consecuencia una serie de medidas dirigidas a la abolición de la discriminación entre unidades económicas de estados nacionales." * La interpretación que probablemente se aproxima más a la nuestra es la dada por Myrdal, quien considera que "la economía no se encuentra integrada a menos que todas las vías estén abiertas a todos $\mathrm{y}$ que la remuneración pagada por servicios productivos sea igual, sin tomar en cuenta diferencias sociales, raciales ni culturales." 5 Sin embargo, ésta no es una definición com-

* B. Balassa, "Towards a Theory of Economic Integration", Kyklos, Vol. 14, 1961, pp. 4 y 5. El subrayado es nuestro. De esta cita podemos observar la exclusión que hace Balassa de la integración nacional del concepto de la integración económica, así como la visión altamente mecanicista del proceso, expuesta en la última frase.

5 G. Myrdal, An International Economy, Nueva York, Harper, 1956, p. 11. 
pleta de nuestra propia posición, puesto que también estamos interesados simultáneamente en la ampliación del potencial de cada componente y del conjunto. Algunos economistas pueden estar en desacuerdo con la interpretación que hemos dado al término. Tal vez prefieran hablar, como por lo común sucede, de los efectos positivos y negativos de la integración como si fueran los medios más que las consecuencias de alguna forma de asociación.

En estos términos, Balassa enuncia exactamente su posición al definir integración económica como una situación o proceso de abolición de la discriminación entre unidades económicas de estados-nación. $^{6}$ Al hacer esto ha olvidado, al parecer, que en este proceso pueden surgir formas nuevas y más rígidas de discriminación de facto entre los países y dentro de éstos. Por ello, en el mejor de los casos tales definiciones pueden considerarse tan sólo como esbozos gruesos del tipo de problemas convencionalmente estudiados por los economistas en los territorios metropolitanos. Estos economistas, como Balassa, malinterpretan desde un ángulo de observación privilegiado lo que se denomina "los procesos sociales en el concepto de integración económica". En lo que a ellos respecta, la única razón para su inclusión es su importancia en la destrucción de las "barreras no económicas entre comunidades, razas y estratos sociales". En cuanto a nosotros, no puede existir una teoría de la integración económica satisfactoria que no admita explícitamente la incorporación de las cuestiones y problemas planteados por la transformación política y social.

\section{II}

Además de las cuestiones conceptuales que venimos tratando, quisiéramos dirigir la atención hacia tres omisiones fundamentales en la teoría de la integración económica tal como se ha desarrollado hasta ahora.

En primer lugar, tenemos la noción existente de una teoría positiva de la integración; en segundo término, tenemos la casi exclusiva preocupación por las condiciones que rigen el intercambio de productos, y en tercer lugar se supone que operamos con una estructura de preferencias predeterminada, la cual, a niveles y distribuciones de ingreso establecidas, es esencial para la determinación de los llamados óptimos de bienestar del "más alto nivel". En cualquier teoría significativa aplicable a las economías sobrexplotadas y estructuralmente dependientes, la meta normativa de la integración económica, expuesta en forma explícita, debe ser el logro de la transformación estructural de estas economías, puesto que tal transformación es considerada como la etapa crítica, aunque no la única, en el proceso de aumento de la producción material. Esto significa que los factores dominantes en la integración económica no deben ser las ganancias que deriven de la fusión de los mercados nacionales. Esas ganancias existen y pueden ser de importancia, pero provienen fundamentalmente de las posibilidades de la región y de sus territorios integrantes de acercarse más a los límites de sus fronteras reales de producción y de ampliar los lími-

6 Teoria de la integración económica, México, UTEHA, 1964, y loc. cit. 
tes de sus fronteras de consumo por la competencia y la eficiencia en el intercambio de bienes, entre un grupo de países y dentro de una región. Para nosotros, la mayor ventaja radica en asegurar y promover la integración de la base de recursos de una región y la consecuente posibilidad que esto crea para una ampliación de la frontera de producción de la región y de los países miembros. ${ }^{7}$

$\mathrm{Al}$ escoger las transformaciones estructurales como los factores clave en el proceso de integración, no pretendemos negar la existencia de diferencias en las valoraciones subjetivas que las personas hacen de las metas normativas del crecimiento. Nuestra elección surge de dos consideraciones básicas que creemos tienen importancia fundamental desde un punto de vista político, económico y social (ambas serán examinadas en la sección siguiente). Una de éstas se deriva de la sobrexplotación o dependencia estructural "excesiva" de muchas de las economías del Tercer Mundo con respecto a los mercados de las naciones industrializadas. La otra se manifiesta en el patrón de industrialización y en los tipos de políticas introducidos $\mathrm{y}$ adoptados por muchas de las economías del Tercer Mundo a través de los dos últimos decenios. Baste decir por el momento que nuestro razonamiento conduce a la conclusión de que la transformación estructural, según la interpretación aquí dada, es el estrangulamiento material crítico de su proceso actual de crecimiento.

Interpretamos la transformación estructural como un aumento continuo de la dependencia intersectorial, tanto en la economía regional como en sus unidades integrantes. Tal dependencia creciente está subordinada a la interconexión entre la estructura de la producción y la del consumo. En este sentido amplio, se ha establecido una relación inversa entre la capacidad para transformarse estructuralmente y el tamaño físico de un país. ${ }^{8}$ En gran parte de la literatura económica se supone que mientras más grandes sean un país o una región, mayor será la probabilidad de que posea una dotación de recursos diversificada. También se supone que la proposición contraria sea cierta. Este supuesto ha conducido a la tesis importante, aunque desalentadora, de que para las pequeñas áreas, como el Caribe y Centroamérica, puede ser imposible desarrollarse hacia una economía madura aun a través de la integración, en el sentido de que sus partes integrantes no pueden crecer proporcionalmente. Esto se revela estadísticamente por coeficientes muy bajos o de cero en la matriz insumoproducto de la región.

Disentimos de esa opinión. Su premisa fundamental es que los materiales básicos en el consumo humano son usados bastante generalizadamente como insumos en cada actividad e influyen de manera bastante uniforme en la ponderación del consumo total. ${ }^{9}$ Pero aun la

7 En forma separada se analiza el papel desempeñado por la agricultura en el proceso de transformación estructural. No existe una necesidad analítica para hacerlo así, sino que sentimos que con ello podremos hacer énfasis en el olvido a que ha estado sujeto este sector en la teoría existente.

8 Charles P. Kindleberger, Foreign Trade and the National Economy, Yale University Press, 1962; William G. Demas, The Economics of Development in Small Countries with Special Reference to the Caribbean, Montreal, McGill University Press, 1965.

9 Esto se puede medir estadísticamente por el grado de dispersión de los coefi- 
revisión más superficial de las matrices de insumo-producto de las llamadas economías maduras revelaría lo incorrecto de tal supuesto. Se observará que en realidad son dos los materiales básicos -hierro y acero, y textiles-, los que por sí solos constituyen la espina dorsal del consumo industrial. En general, a pesar de la multiplicidad de productos finales, es posible alcanzar un grado elevado de madurez económica industrial con la producción de una variedad bastante reducida de materiales. Estos productos son papel, plásticos, hule, vidrio, cuero, cemento, madera, combustibles, productos químicos industriales (principalmente los álcalis, el cloro y el ácido sulfúrico) $\mathrm{y}$, por supuesto, hierro y acero, y textiles.

Un testimonio notable de nuestra proposición estaría dado por un estudio atento del proceso de industrialización experimentado por países físicamente tan pequeños como Gran Bretaña o aun Bélgica. Esta proposición tiene la implicación ulterior de que algunos países físicamente pequeños puedan tener, vistos desde el lado de la oferta -digamos, aquellos con reservas de hierro, carbón y petróleo y materiales fibrosos-, capacidad para un desarrollo industrial de grado bastante maduro. Mientras que, por otro lado, es posible que países físicamente de gran tamaño, o aun continentes, puedan estar severamente constreñidos o encontrar ese desarrollo en exceso costoso porque, a pesar de la diversidad de sus recursos, puedan resentir deficiencias correspondientes a los bienes de consumo más críticos. En una clasificación gruesa podríamos catalogar como ejemplos de la proposición inicial a territorios tales como las Rhodesias y Chile, y a Argentina como ejemplo de la última.

Si bien a través de este documento hacemos mayor énfasis en los beneficios derivados de la integración de la utilización de los recursos, sigue siendo cierto, no obstante, que la madurez industrial del tipo que estamos examinando no se logra independientemente de la estructura de la demanda. Desde luego que son de importancia tanto los patrones de crecimiento y las estructuras de la demanda como los de la oferta, como lo es por ende la política comercial. Un país puede aparentar un grado elevado de madurez industrial si el patrón de su demanda se ha hecho coincidir, en mayor o menor grado, con aquellos materiales básicos y otros elementos estratégicos de su eslabonamiento interindustrial que es capaz de producir. Existen otras manifestaciones de atraso y madurez económica, pero la simetría en el desarrollo estructural corresponde a nuestra noción de una economía en evolución hacia la madurez.

Más precisamente, nuestra perspectiva del proceso de desarrollo prevé que el grado de transformación estructural se mide a través de la brecha entre la estructura y el patrón de la demanda interna y la estructura y el patrón de la producción interna. Esta brecha puede no mostrarse en forma adecuada a través de las propensiones a importar tradicionales (relación entre importaciones y producto bruto). Si una proporción significativa de las importaciones constituye tan sólo un insumo de exportación y el bien de exportación no se consume inter- 
namente, las elevadas propensiones resultantes tenderán a ocultar desarrollos significativos en la economía interna. El grado de transformación estructural tampoco se mide por medio del coeficiente de importación (relación entre importaciones y gasto u oferta totales). Este coeficiente incluye importaciones tanto en el numerador como en el denominador y, según sea el tamaño relativo del sector interno, puede resultar inadecuado. Tampoco resulta satisfactorio un coeficiente en términos de contenido de importaciones, debido a que la producción para el consumo interno puede ser igual a apenas una porción del gasto interno.

Proponemos que estos conceptos sean remplazados por uno nuevo denominado coeficiente de importación sobre gasto interno, que relacione el valor y el patrón de las importaciones para uso interno con el valor y el patrón del gasto interno. Y puesto que estamos midiendo el grado de transformación estructural en términos de la brecha entre el patrón y la estructura de la producción interna y el patrón y la estructura de la demanda, esto implica que pueden existir diferencias significativas ocultas en una comparación simple del contenido de importaciones de las exportaciones y el contenido de importaciones del gasto interno. Así, en el caso de un sector exclusivo de exportación desproporcionadamente grande, que derive sus insumos casi absolutamente de las importaciones, existirá una divergencia aguda entre la propensión a importar y el coeficiente de importación sobre gasto interno. Como señalamos antes, el primero no sólo tiende a proporcionar un cuadro deformado de lo que ocurre en la economía interna, sino que lleva a comparaciones bastante engañosas con los países en donde las divergencias entre la estructura de la producción y de la demanda son menos radicales, aun cuando estos paises puedan tener ingresos per capita más bajos.

No debe extrañar que el concepto introducido no ocupe un lugar prominente en una literatura económica dominada por economistas de los países "maduros". En estos territorios la producción y la demanda generalmente coinciden. La especialización en la exportación, cuando existe, surge casi invariablemente como extensión de la demanda interna. Esto es distinto a lo que ocurre en muchas de las economías del Tercer Mundo en donde, por el proceso de colonización, la especialización en exportaciones está regida por las demandas de los mercados metropolitanos.

Otra característica, algunas veces utilizada para describir el proceso de transformación estructural y económica y que puede interpretarse como similar a nuestro concepto, es la relativa a la disminución de las diferencias intersectoriales de productividad del trabajo. Nuestro concepto de transformación estructural no significa necesariamente esto, pues podría entonces dar origen a una interpretación demasiado restringida de la naturaleza de la maduración económica. Tal vez a este respecto sería pertinente señalar algunas de sus limitaciones. La primera de éstas es obvia en situaciones donde las economias acusan dualismo tecnológico. Así, por ejemplo, el sector minero explotado directamente, que emplea por lo general un número reducido de personas, puede absorber una proporción muy reducida de la fuerza de trabajo total, mientras que su producción por hombre es varias veces 
superior a la de otros sectores explotados indirectamente. Una posible corrección estadística consiste en obtener la desviación media de las productividades intersectoriales ponderadas por el empleo relativo del sector. Pero nuestra objeción a este concepto es más fundamental.

Los diferenciales de productividad del trabajo no coinciden necesariamente con otros diferenciales de productividad, v. gr. del capital, la tierra o aun la productividad de "todos los factores". Existen tasas diferenciales de insumo de los factores en una actividad, así como diferentes tasas de crecimiento de las diversas actividades de la economía. Para efectos de establecer criterios en cuanto a la transformación estructural no existe razón para que el denominador de la productividad sea necesariamente el insumo-trabajo. Este criterio tiene especial importancia en zonas de supuesta escasez de capital y en donde, por la naturaleza de la tecnología, el capital sea el insumo intensivo.

Los economistas que interpretan el proceso de transformación estructural en términos de la reducción de las diferencias de productividad del trabajo, estiman que dicho proceso connota un movimiento hacia una mayor eficiencia económica general en el sentido de igualar costos marginales. Esta premisa, que corresponde a otros aforismos neoclásicos, es desde luego insuficiente teóricamente para tal resultado. Pero la idea en sí de igualar costos marginales, como criterio de un proceso dinámico de transformación estructural, aun al nivel puramente económico, está sujeta a serias dudas. En todo momento se puede decidir que los beneficios resultantes de una mayor eficiencia pueden asumir una importancia menor que los obtenidos del crecimiento en sí mismo de la producción. No siempre se pueden hacer coincidir las consideraciones relativas a eficiencia con las de expansión.

Esta discusión sobre la transformación estructural sirve para dos propósitos, a saber: i) esclarecer el significado económico del concepto y ii) indicar que la integración económica debe establecer como su meta material primaria la transformación estructural en el sentido expuesto. El siguiente problema que afrontamos se refiere a por qué la transformación estructural se podría convertir en el estrangulamiento crítico del proceso de crecimiento. Intentaremos dar una respuesta breve refiriéndonos a dos factores, a saber: i) la naturaleza de las economías estructuralmente dependientes y ii) las limitaciones a los actuales procesos de industrialización en muchas de las economías del Tercer Mundo.

\section{III}

En aras de la brevedad apenas enumeraremos algunos de los rasgos sobresalientes de las economías estructuralmente dependientes. Muchos de ellos son bien sabidos por la mayoría de los economistas, pero los destacaremos aquí principalmente con objeto de proporcionar el trasfondo de su desarrollo. Desde el enfoque dado a nuestro análisis se sigue naturalmente que el indicador particular de mayor importancia de la dependencia estructural es la brecha entre el patrón y la estructura de la producción interna y el patrón y la estructura de la utilización interna. Los coeficientes típicos de importación sobre gasto interno son elevados y pueden variar entre 0.30 y 0.70 . Las pro- 
pensiones a importar y los coeficientes de importación son también muy altos, lo que indica que en general la producción se encuentra especializada en ventas para la exportación. Estas ventas para la exportación no constituyen una forma de compensar el tamaño reducido del mercado interno. Son un resultado característico de la explotación colonial de los recursos locales para uso de las metrópolis.

$\mathrm{Al}$ ser productores altamente especializados, las proporciones de exportación de estas economías son también muy elevadas. Sin embargo, a esta característica se agregan cuatro defectos adicionales. El primero es que la producción para la exportación (casi igual a la producción total) es esencialmente de materiales primarios, bien sea materias primas agrícolas o productos minerales tales como bauxita y petróleo crudo. Esto significa que su impacto generativo en el desarrollo de la economía interna es virtualmente cero. El segundo, que la producción para exportación se concentra en una variedad limitada de bienes, generalmente dos o tres. De este modo, si tomamos un ejemplo de un territorio de este tipo, digamos Guyana, las exportaciones de azúcar, bauxita y arroz constituyen por sí solas cerca del $75 \%$ del total de las exportaciones, a la vez que las exportaciones totales representan entre el 55 y el $60 \%$ del ingreso nacional. El tercer defecto radica en que las ventas de estos bienes están fuertemente restringidas a unos cuantos mercados. Así, nuevamente en el caso de Guyana, cerca del $75 \%$ de las exportaciones se destina al Reino Unido y a Norteamérica. El cuarto factor está representado por la considerable inestabilidad que al volumen y al precio de los productos agrícolas y minerales de exportación ocasionan las condiciones de la oferta y la demanda mundiales. En las economías estructuralmente dependientes el grueso de la inversión de capital se canaliza hacia el sector de exportación y a su vez es suministrado principalmente por ahorros del exterior. En estos términos, una relación característica entre inversión extranjera e interna es de $70: 30$.

Huelga decir que estas economías son pobres en términos del más convencional de los indicadores: el bajo ingreso per capita. Siendo crítica en sí misma, esta situación se complica aún más por dos factores: un grado pronunciado de dualismo tecnológico donde los sectores de plantación y minero no sólo emplean la mejor tecnología en uso sino que también pagan tasas de salarios superiores a los costos sociales "reales" del trabajo. Este último factor significa una fuerte división del mercado de trabajo, siendo la prosperidad de los sectores de salarios elevados el elemento clave en la determinación de las retribuciones al factor trabajo por su contribución a la producción nacional. Esta situación se acompaña generalmente de un nivel elevado de desempleo y subempleo. En realidad es de tal magnitud, entre el 18 y el $25 \%$, que para muchos territorios se convierte en el problema económico y social particular más urgente. En términos económicos, significa que el costo "real" del trabajo difícilmente debería estar por encima del nivel del salario de subsistencia, y en este sentido el sector moderno de salarios elevados se convierte en un ejemplo de la distorsión del mercado de trabajo.

Existe gran número de otras pruebas de la explotación, la dependencia estructural y la inmadurez económica. Algunas serán señaladas 
más adelante; por ahora podemos apuntar el hecho de que los convenios comerciales y financieros de estas economías dependen en forma considerable de los mercados de dinero y las instituciones financieras de las áreas metropolitanas. No sólo muchos de los bancos de estas zonas son sucursales de bancos metropolitanos, sino que con frecuencia las leyes locales exigen que la liquidez interna esté estrechamente vinculada a la adquisición de divisas extranjeras. Esto significa que cualquier ahorro que pase por el sistema de intermediación financiera tiene elevada propensión a ser invertido fuera del país. ${ }^{10}$ Desde luego que esta lista de defectos puede multiplicarse varias veces, pero para nosotros basta con lo dicho para poder apreciar el fondo de las políticas de desarrollo en estos territorios.

En el período inmediato de posguerra surgió una serie de actitudes conflictivas hacia los problemas planteados por las economías estructuralmente dependientes. En un principio, existía la creencia generalizada y aun oficial de la impractibilidad económica de la industrialización de estas zonas (muchas de ellas colonias), y en cierta forma se sentía que esto era en todo caso indeseable. Esta posición estaba de acuerdo con la interpretación metropolitana de los "hechos" y tendía también a coincidir con un período de desempleo relativamente bajo (debido a las presiones posbélicas para expandir la producción) y de precios favorables de los productos básicos (motivados por las escaseces de la posguerra). La respuesta a esta situación por parte de los inversionistas fue también acorde con la opinión oficial. De este modo se tomaron medidas rápidas tanto para ampliar la superficie de labor destinada a la producción primaria, como para mecanizar la producción con miras a obviar la falta de mano de obra.

Esta posición había cambiado a principios de los años cincuenta. El surgimiento de políticas y actitudes nacionalistas, el rápido incremento demográfico, las políticas de mecanización excesiva de la agricultura de plantación y el relativo estancamiento de los ingresos llevaron a considerar la industrialización como única solución. Esto coincidía con la noción "ilustrada" que del desarrollo económico se adquirió en las metrópolis. Como política fundamental para hacer frente a este desafío, se elaboró toda una serie de concesiones tendientes a inducir al capital extranjero, y en menor grado al local, a efectuar inversiones en la economía local. El patrón y los problemas de este tipo de industrialización son los que ahora nos traen a examinar los límites que plantean al proceso de crecimiento.

En un principio, estas políticas, basadas en la malentendida experiencia de Puerto Rico y en interpretaciones espurias de la industrialización japonesa, tuvieron la apariencia de ser notablemente exitosas. A partir de los años cincuenta se hicieron adiciones sustanciales a las industrias manufactureras. La producción industrial acusó una tasa elevada de crecimiento pero, por partir de niveles bajos de desarrollo manufacturero, los incrementos absolutos no fueron significativos. De este modo, Jamaica y Trinidad-Tobago, donde el crecimiento de la producción industrial excedió con mucho el promedio de los países en

10 Véase Clive Thomas, Monetary and Financial Arrangements in a Dependent Economy, Kingston, Jamaica, Institute of Social and Economic Research, University of West Indies, 1965. 
desarrollo, se encontraron con que en realidad esta producción excedía apenas ligeramente la tasa de crecimiento de su producto bruto. En estos términos, el valor de su producción manufacturera representa aún tan sólo el $10 \%$ del producto bruto interno.

De mayor significación es el tipo de industria que se ha establecido. Debido al tamaño reducido de los mercados nacionales, la mayor parte son industrias de ensamblado y acabado. En promedio, el valor bruto de las adiciones internas a la producción es de menos del $20 \%$, representado casi por completo por costos de trabajo. En esta situación, el atractivo para el inversionista, extranjero o local, es la posibilidad de que las concesiones gubernamentales pudiesen conferir un monopolio virtual a sus empresas, $y$ en realidad nuestro estudio del Caribe sugiere que éste es el factor particular más importante subyacente en el proceso de industrialización. ${ }^{11} \mathrm{El}$ resultado neto de estas políticas es la casi inexistencia de eslabonamientos hacia atrás o hacia adelante entre cualquiera de las actividades manufactureras existentes. Casi no se han hecho intentos para integrar los materiales locales al proceso manufacturero. La forma común de racionalizar esta deficiencia ha sido a través de la aceptación demasiado fácil de la afirmación relativa a la escasez o inexistencia de recursos industriales básicos. El Caribe es tal vez el mejor ejemplo del abuso de la comunicación científica metropolitana. ${ }^{12} \mathrm{~A}$ pesar de las rápidas tasas de industrialización, la brecha entre la estructura de la producción y la estructura de uso interno sigue siendo tan amplia como antes, si no es que más extensa.

Existen algunas desventajas específicas creadas por el patrón y el enfoque actuales de la industrialización. En primer lugar, se inhibe la investigación tecnológica local al no emplearse casi nunca los materiales locales en algún proceso integrado internamente que llegue hasta la producción final. La inversión se efectúa bien sea en materiales primarios para la exportación (el patrón tradicional) o en el ensamblado y acabado de artículos manufacturados producidos casi por completo en el exterior. El enfoque de la industrialización como hasta ahora se ha presentado no ha dado una "prominencia explícita al contenido de recursos de los proyectos industriales".'13 Al nivel de la teoría del desarrollo tiene carácter convencional postular que el capital, las divisas extranjeras y la tierra constituyen recursos escasos mientras que otros, en particular el trabajo, dadas las altas tasas de desempleo, son abundantes. El problema de optimización se convierte entonces en un problema de minimización de los coeficientes de los factores escasos.

Un ejemplo clásico de este enfoque teórico es el sugerido por el profesor Lewis. ${ }^{14} \mathrm{Al}$ aplicar estos criterios de optimización (minimizar los coeficientes de insumos escasos y maximizar los coeficientes de

$11 \mathrm{H}$. Brewster y C. Y. Thomas, op. cit., cap. 3.

12 H. Brewster, Resource Use and Priorities in Caribbean Industrial Development. Este trabajo fue presentado en la Conferencia de Tecnología y Desarrollo en Sociedades Transicionales Pequeñas, University of West Indies, Trinidad, febrero de 1968.

13 Ibid.

14 W. A. Lewis, The Industriatization of the British West Indies, San Juan, Puerto Rico, Caribbean Commission, 1950. 
trabajo), Lewis encontró que las industrias más desfavorables para el Caribe serían las siguientes: fertilizantes, molinos harineros, leche evaporada, margarina, cemento, loza, cerveza, alimentos para ganado, jabón, pinturas y refinación de petróleo. Pero a partir de entonces, i todas estas industrias se han establecido en diversos territorios del Caribe! Igualmente, de su lista de industrias más favorables, o sean conservación de pescado, tejidos de punto, relojes, paraguas, escobas y cepillos, artículos para deporte, tejidos y lámparas eléctricas, ¡ninguna ha sido establecida con firmeza en ninguno de los territorios de la región! En todo caso, el éxito de su lista apenas pudo haber sido superior al de lo ocurrido en realidad. La razón de lo anterior es que lo "desfavorable" para Lewis se podría interpretar en realidad como "improbable" más que como "indeseable".

La transformación exitosa de esta región requiere de una estrategia explícita de desarrollo que dé preeminencia a dos criterios. Primero, se deberá dar prelación al contenido de recursos locales de todos los proyectos industriales. Lo anterior es consecuente con nuestra interpretación general de la maduración económica (transformación estructural) expresada arriba. En la utilización de los recursos locales se deberá dar énfasis a aquellos recursos limitados que sean utilizados en forma predominante en la elaboración de toda la producción manufacturera final -hierro y acero, textiles, papel, plásticos, hule, vidrio, cuero, cemento, madera, combustibles y productos químicos industriales. El otro elemento que debería tener alta prelación es el potencial bastante grande de demanda dinámica que existe en la mayoría de estas economías para ciertas categorías de bienes, por ejemplo, algunos tipos de comestibles, principalmente los de alto contenido proteínico $\mathrm{y}$ con fuerte preferencia en los gustos (es decir, carne, legumbres, productos lácteos), y otros artículos, como automóviles y turismo. Estos artículos tienen un potencial de demanda elevado en el sentido de que las elasticidades-ingreso de la demanda son altas, a la vez que, en general, los factores que acompañan al desarrollo tales como la urbanización, la expansión de los medios de comunicación, etc., producen un fuerte sesgo de "gustos" en esa dirección.

Este enfoque del desarrollo permite también considerar el problema de la utilización del trabajo en condiciones mejores que conforme a la práctica existente. Tomemos un ejemplo del que aparentemente es el "peor" de los casos, esto es, las llamadas industrias básicas e intermedias de alta intensidad de capital. Debido a que el número absoluto de personas en estos territorios "pequeños" es también pequeño, el efecto-empleo de una industria básica en gran escala es relativamente grande. Así, por ejemplo, una industria siderúrgica básica de 300000 toneladas de capacidad podría proporcionar empleo a 2500 personas, lo que en una economía como la de Jamaica representa más de la cuarta parte del empleo directo creado en veinte años de industrialización. $\mathrm{Y}$ esto sin mencionar los beneficios que resultarían de los efectos de "eslabonamiento" y otros. Un ejemplo aún más notable sería el empleo directo que generaría la producción de 35000 toneladas de textiles (incluyendo el cultivo del algodón), que sería aproximadamente de 13000 personas. Éstos serían casi tantos como los empleados en la industria petrolera de Trinidad. Dicha industria contribuye aproxima- 
damente con la tercera parte del producto interno al costo de los factores en esta economía cuyo ingreso per capita es el cuarto más alto del hemisferio occidental.

La segunda desventaja específica es aquella que señalábamos antes. La proporción del valor interno agregado por la industria será baja como consecuencia de la desventaja anterior. Además, por la natureleza misma de la organización estructural de la industria, seguirá siendo consistentemente menor a la porción correspondiente agregada en las economías económicamente "maduras". Por lo tanto, es de esperar que la brecha de ingreso entre los países ricos y los pobres no se reduzca. De mayor importancia, algunas de las formas comunes de acercamiento al problema por ejemplo, a través de la liberalización del comercio, no han sido capaces de comprender la naturaleza esencial del fenómeno. El empleo total creado por unidad de producto final será también correspondientemente menor, lo que, además del dualismo tecnológico, explica en parte la reducida capacidad de absorción de trabajo de estas economías. En tercer lugar, la localización industrial se ha concentrado por lo regular en las ciudades que parecen estarse industrializando a tasa elevada. En cuarto lugar, el patrón y el proceso de industrialización han tendido en su conjunto a oscurecer el significado de las economías de escala y de este modo han proporcionado una racionalización para el actual proceso de industrialización, defectuoso como es. En el ensamblado final, las economías de escala son bastante insignificantes si se comparan con los ahorros obtenidos a través de las economías dimensionales de los procesos de elaboración de materiales básicos e intermedios como los mencionados anteriormente.

Por último, estas políticas han propiciado la migración del campo a la ciudad. Esto ha sido motivado por diversos factores. En primer término, como es sabido, en muchos de estos territorios existe un desajuste continuo y bastante amplio entre la absorción anual total en el empleo y el incremento anual de la fuerza de trabajo. Esta situación se ve agravada por otro desajuste que comúnmente surge de la mayor tasa de incremento natural de la población rural en comparación con la de la población urbana. Este diferencial, aun bajo políticas de localización industrial menos sesgadas, tendería a causar desequilibrios demográficos entre diversos distritos. En segundo lugar, ha ocurrido un deterioro continuo de la relación de precios del intercambio cle bienes entre la ciudad y el campo y esto ha alentado la emigración rural. Los incrementos de la productividad aceleran este movimiento de dos maneras. Por una parte, al generar una caída de los precios relativos, la relación-ingreso del intercambio puede no mejorar, y en ocasiones hasta puede decaer, y en realidad, la idea de un crecimiento pauperizante va dejando de ser una curiosidad teórica al aplicarse a las transacciones interregionales en lugar de las internacionales; más aún, algunos de los aspectos más importantes del crecimiento pauperizante deberán encontrarse en el creciente desempleo y en la creciente desigualdad de la distribución del ingreso, factores que no forman parte del análisis teórico de este concepto. ${ }^{15}$

15 J. Bhagwati, "International Trade and Economic Expansion", American Economic Review, diciembre de 1958. 
Por otro lado, el incremento de la productividad rural en el sector de plantación también acelera la migración rural-urbana al reducir la cantidad de insumo de trabajo por unidad de producto. Esto seguiría ocurriendo a menos que la tasa de incremento de la producción real fuera mayor que la suma de las tasas de incremento de la productividad y de la fuerza de trabajo. Se puede apuntar aquí en forma colateral que esto podría proporcionar una racionalización económica (poco convencional, desde luego) de la "aparente" renuencia de la comunidad agrícola campesina a elevar la productividad o aumentar en mayor medida la producción.

En consecuencia, las ciudades se convierten cada vez más en núcleos de pobreza aguda, desigualdad conspicua y tensión social. Así pues, al examinar el concepto de integración económica argumentábamos que esta situación socialmente polarizada y desintegrada de la economía nacional planteaba problemas adicionales a la integración funcional de una región. Como se expone en la teoría de Myrdal, estas cuestiones se convierten en una preocupación integral y legítima de cualquier intento de síntesis económica.

En términos dinámicos, la relación entre trabajo, productividad y necesidades de capital constituye un problema decisivo para las áreas estructuralmente dependientes.16 $\mathrm{La}$ tasa de absorción de la mano de obra es función de dos variables principales: la tasa de incremento de la producción y la tasa de aumento del producto por hombre. Por lo tanto, la tasa de incremento de la producción real menos la tasa de incremento de la productividad del trabajo equivale aproximadamente a la tasa de absorción de la mano de obra. Si, como parece ocurrir en muchas de estas economías, la oferta de mano de obra aumenta cerca del $3 \%$ anual y la productividad del trabajo se incrementa un $6 \%$ anual, la producción real para absorber solamente las adiciones anuales de la fuerza de trabajo debería aumentar en cerca del $9 \%$ al año. Dado el tipo actual de desarrollo, el sostenimiento de un crecimiento tan elevado de la producción real necesitaría de niveles de inversión que rebasan la capacidad actual de estas economías (incluida su capacidad para atraer capital extranjero). En el estudio señalado, la CEPAL plantea el problema de la siguiente manera: "Es en gran parte... [el] desequilibrio entre productividad e inversiones... Es cierto que el aumento de ingreso proveniente del incremento de productividad genera mayor capacidad de ahorro. Pero el capital requerido para la mano de obra redundante - provocado por esa mayor productividad - es superior al ahorro que momentáneamente pudiere obtenerse; y sólo con el tiempo podrá lograrse el equilibrio entre la mayor acumulación de capital exigida por el incremento de productividad y la mayor capacidad de ahorro que ésta trae consigo. Es, pues, un desequilibrio temporal, pero de gran significación..."

En principio, la literatura económica contemporánea sugiere tres formas de aproximarse en forma positiva a esta cuestión, a saber: 1) aumentar la tasa de ahorro para hacer frente al desequilibrio; 2) re-

16 Raúl Prebisch, Hacia una dinámica del desarrollo latinoamericano (CEPAL, Doc. E/CN.12/680), México, Fondo de Cultura Económica, 1963, especialmente pp. 25-52. 
ducir la tasa de productividad del trabajo y 3 ) reducir la tasa de aumento de la fuerza de trabajo.

Nuestra teoría conduce a una cuarta posibilidad: la expansión del horizonte espacial de un país individual ligada estrechamente a la integración de la base de recursos de la región ampliada. Esto permitiría la expansión de la capacidad de la región y del territorio para producir materiales básicos, comestibles y otros componentes estratégicos del consumo humano y con esto reducir la brecha entre la estructura de la producción y de la demanda internas. Con objeto de asegurar lo anterior, es necesario que fluyan fondos disponibles para inversión hacia esos sectores, puesto que, sobre todo en la agricultura, su capacidad de absorción es considerable.

Por lo tanto, en esta cuarta alternativa ponemos en duda en forma explícita la premisa de la cual se derivaron las respuestas teóricas tradicionales. Al hacerlo así estamos sugiriendo de hecho que el problema al parecer insoluble planteado por el desequilibrio entre las necesidades de capital y la productividad, que acabamos de explicar, está condicionado a su vez por la trayectoria de desarrollo que siguen muchos países. Esto no se refiere únicamente al tipo de tecnología que se está empleando. En este sentido son poco afortunadas la excesiva concentración en la intensidad de capital de la tecnología usada y en cierto grado también la concentración sobre el control demográfico. Esto se debe a que, en esencia, son críticas implícitas a acontecimientos que, si se pudiera desacelerarlos, servirían apenas para una leve mejoría de las condiciones existentes en los países de menor desarrollo. Nuestra cuarta alternativa procura, por lo tanto, enfatizar el efecto generativo de un tipo de desarrollo orgánicamente estructurado que se hace posible a través de la integración de sistemas económicos. Entre los diversos factores involucrados en este proceso uno de los principales es el reajuste estructural de las instituciones comerciales y financieras con objeto de: a) reducir la emigración de ahorros internos y $b$ ) evitar la canalización errónea de fondos para inversión. Existe mayor posibilidad de que esto suceda aunque sólo sea porque la diversidad de las oportunidades regionales es preferible a una nación de tamaño limitado.

Al concluir esta parte del trabajo podríamos sintetizar de la manera siguiente. El actual proceso de industrialización en las economías estructuralmente dependientes se encuentra limitado en su capacidad para reducir la brecha entre la estructura de la producción y la demanda interna, para aumentar la proporción de transacciones intersectoriales, para facilitar encadenamientos hacia adelante y hacia atrás y para usar los materiales locales. La integración económica regional proporciona la mejor alternativa para superar estos estrangulamientos en el proceso de crecimiento. Sin embargo, en cualquier integración de este tipo sería necesario poner énfasis en la coordinación de la planeación y la producción. La promoción deliberada de una variedad suficiente de industrias de materiales básicos y de cierto tipo de comestibles (que se mencionarán posteriormente) se convierte en la primera prioridad en el camino hacia la madurez económica. También se convierte en la primera tarea de las autoridades regionales. Las condiciones que rigen el intercambio de bienes existentes o de bienes con 
probabilidades de ser producidos bajo los ímpetus e iniciativas existentes deberán desempeñar un papel subsidiario.

\section{IV}

En la teoría de la integración económica actual apenas si existe consideración alguna del papel de la agricultura. Desde nuestro punto de vista esto no puede ser así. La integración agrícola es crítica desde el punto de vista del equilibrio estructural, y de los cambios políticos y sociales. En muchas de las economías estructuralmente dependientes la agricultura proporciona la mejor alternativa para absorber el excedente de trabajo. Como ya se señaló, la producción de una economía estructuralmente dependiente está especializada. Aun cuando la economía produzca en lo principal bienes agrícolas (que es el caso de muchas de estas economías), esa producción se limita en forma importante a la exportación de materias primas. La demanda de alimentos de la población local (y del creciente número de turistas) se cubre mediante importaciones. La reducción de la brecha entre las estructuras de la producción y de la demanda requiere de la producción de un porciento elevado de los comestibles por ahora importados. Esto es realmente deseable desde otro aspecto puesto que el potencial de demanda dinámica y las proporciones entre recursos naturales, tierra, trabajo y capital utilizadas en la producción de estos comestibles importados son favorables a las ventajas que proporciona la flexibilidad regional en el patrón de uso agrícola de la tierra.

En la teoría de la integración económica la ampliación del mercado conduce a los clásicos "beneficios del comercio". En el caso de la agricultura en las economías estructuralmente dependientes esto se presentaría bajo condiciones de "desviación del comercio". Para nosotros, los beneficios reales no pueden surgir en forma natural de la estructura de la producción actual, sino que deberán ser resultado directo tanto de una política pública deliberada que pretenda lograr por el lado de la oferta una combinación de los recursos agrícolas de la región, como de la protección habitual que asegure la desviación del comercio. Por lo tanto, las ganancias de la integración económica en la agricultura sólo se podrán apreciar a través de evaluaciones comparativas de una política de sustitución de importaciones regional frente a una de sustitución de importaciones nacional.

En la teoría tradicional de la integración, la competencia entre áreas especializadas en la producción de cosechas de exportación produce beneficios a estas zonas sólo en la medida en que a) parte de su producción se consuma localmente, y b) los mercados en los países metropolitanos sean tales que confieran algún grado de protección a los productores de la región con costos relativamente altos. Si en el período previo a la integración existiera un territorio en la región que produjera a costos relativamente altos, su supervivencia durante el proceso de integración dependería del grado en que fuera protegido de la competencia mundial en los mercados metropolitanos y/o de si el área productora con costos relativamente bajos no pudiera producir las cantidades del bien requeridas en los mercados metro- 
politanos. Si se mantiene esta condición, es posible teóricamente que la integración y la competencia incrementada en la zona puedan llevar a un aumento del bienestar y de la eficiencia en el sentido de Viner. Esto se daría a través de una disminución del precio de la parte de la producción que se consumiera dentro de la región, y a través de un cambio de una fuente de oferta interna con costos relativamente altos a una de costos más bajos.

En las economías estructuralmente dependientes dichas ganancias tal vez tengan carácter marginal porque la proporción de bienes de exportación consumidos internamente es reducida. En segundo lugar, la disponibilidad de tierra puede hacer que no sea posible efectuar volúmenes importantes de "sustitución de exportaciones". En tercer término, si la sustitución de exportaciones se lleva a cabo, significa que partes de la región se están volviendo aún más dependientes estructuralmente, en la medida en que aumenta la proporción de tierra y otros recursos que fluyan hacia la producción de cosechas especializadas.

Por lo tanto, volviendo a nuestra proposición concerniente a los méritos superiores de un enfoque agrícola regional, podemos plantear la siguiente pregunta: ¿en qué dirección surgen estas ganancias? Un mercado regional para la producción agrícola interna tiene muchas ventajas además de las que se originan en los mercados nacionales. En primer lugar, es probable que suceda lo que se puede describir como efecto desplazamiento, o efecto-impacto de una vez por todas en la producción agricola. Esto se refiere al estímulo que probablemente se daría a cualquier sector de cualquier economía como resultado de alguna forma de integración del mercado. Llevaría a un incremento más o menos garantizado, grande, concentrado e independiente en el tamaño del mercado para la producción del sector. Estas ventajas se pueden manifestar de diferentes maneras; por ejemplo, mejoras en los controles de calidad y clasificación de la producción, de gran importancia en la agricultura, que parecen estar asociadas a la exigencia de servir a mercados más grandes, ya sea en volumen de ventas o por distancias económicas. O bien estas ganancias pueden expresarse en términos de la confianza y la estabilidad de expectativas que la integración puede originar en los productores. Dichas ganancias pueden ser significativas para las economías estructuralmente dependientes puesto que han sido explotadas en el pasado por las llamadas economías maduras a través de la inestabilidad estructural inherente a las ventas de bienes a ultramar.

En segundo lugar están, por supuesto, los tradicionales efectos-precio e ingreso en el consumo que aparecerían por la fusión de mercados. En tercer lugar, la evidencia empírica muestra que la demanda de productos que sustituyan importaciones, tales como mantequilla, queso, legumbres, carne, etc., pueden tener un potencial dinámico sumamente elevado. Claro que se podría argumentar que estas ventajas existen también a nivel nacional, pero desde el punto de vista de la región tanto ésta como sus territorios podrían beneficiarse en la medida en que los coeficientes combinados ponderados de las actuales elasticidades-ingreso y otros factores de la demanda dinámica sean superiores a los existentes comúnmente en cualquier 
territorio individual. Dicho en otra forma, los beneficios serán obtenidos en la medida en que no exista simetría completa entre el potencial de producción dinámico y el potencial de demanda dinámico en cada territorio. Estas ganancias se obtendrian aun cuando la tasa de crecimiento de una región integrada no excediese el promedio ponderado de la tasa obtenida generalmente en la zona.

Por último, bajo las ganancias de mercado es de esperar que si la integración conduce a crecimiento y desarrollo generales más rápidos en la región (lo que creemos sucedería), entonces las ganancias dinámicas serán aún mayores que las mencionadas arriba. Aumentarán más rápidamente cuanto más elevada sea la tasa de crecimiento y desarrollo regional de la región por encima de la tasa resultante de la suma de las tasas de crecimiento de los territorios separados no integrados. Esta consideración es de importancia crítica puesto que las tasas de crecimiento de estas economías proyectadas con bases separadas no son suficientes para absorber los incrementos anuales de la fuerza de trabajo, y mucho menos el desempleo acumulado.

Un incremento del tamaño del mercado puede por sí mismo llevar a ganancias dinámicas de eficiencia por medio de la reducción de los coeficientes de insumo, aun con la disponibilidad de recursos existente. Las ganancias estáticas clásicas de la eficiencia se obtienen cuando la región es capaz de operar en las fronteras de su curva de posibilidades de producción. La competencia y las desviaciones planeadas en la localización de la producción, dirigidas a tomar en cuenta las dotaciones agrícolas diferenciales de la región, pueden conducir a cada territorio más allá de sus actuales fronteras de producción. Además, podríamos argumentar que un mercado más amplio en sí y por sí solo puede llevar a incrementos en la productividad por encima de los debidos a los cambios de localización de la producción, el progreso tecnológico, etc. En varios estudios empíricos se muestra una fuerte correlación entre el tamaño del mercado y los cambios en la productividad. ${ }^{17}$ La mayoría de estos estudios se refiere a industrias manufactureras y hasta hay quien sugiere que la productividad se incrementa aproximadamente como función raíz cuadrada del volumen de la producción. ${ }^{18}$

Aunque pudiera pensarse que estos estudios tienen poco interés para la agricultura, no debe olvidarse que muchos procesos agrícolas involucran dimensiones cúbicas; por ejemplo, la crianza de cerdos y la lechería. Las mejoras se aplicarían a aquellas actividades agrícolas que utilizan producción industrial como un insumo importante (por ejemplo, la crianza de los cerdos, en que más del $80 \%$ de los costos se podría atribuir a insumos alimenticios). Sin embargo, la relación quizá sea más compleja de lo que pudiera parecer al principio, y por ello es razonable considerar la relación entre tamaño del

17 Véase D. Paige y G. Bombach, A Comparison of National Output and Productivity of the United Kingdom and the United States (Paris, OEEC); L. Rostas, "Comparative Productivity Levels in British and American Industry", y J. H. Young, "Comparative Economic Development: Canada and the United States", American Economic Review, Papers and Proceedings, mayo de 1955.

18 A. Verdoon, "On an Empirical Law Governing the Productivity of Labour", Econometrica, abril de 1951. 
mercado y productividad como interactuante, en la que cada variable genere cambios en la otra.

El análisis anterior es también válido para el empleo. La tasa de empleo será en parte función de las tasas de crecimiento de la producción y de las técnicas utilizadas en el proceso de producción. De nuevo, la evidencia empírica muestra que en los comestibles importados la relación entre trabajo y producto es más elevada que en la actual agricultura de plantación, particularmente en aquellas zonas (por ejemplo, las de cultivo de caña de azúcar) en que se desplaza con gran rapidez la mano de obra. Si los territorios nacionales desarrollaran mercados de exportación, las ganancias de mercado aumentarían. Pero sería ocioso hacer toda una lista del gran número de factores que impiden a las regiones en desarrollo la venta de sus cosechas en los mercados de los países industrializados.

Del lado de la oferta, existe una serie de economías de escala que se derivarían de la integración económica de la agricultura. En la literatura sobre el tema, esto ha sido descuidado en parte debido a la tendencia a igualar el crecimiento con la industrialización, y también debido a que la escala mínima de producción en la agricultura es muy pequeña ya que, en última instancia, en la práctica sólo se encuentra limitada por las necesidades de supervivencia de un campesino. Sin embargo, existen economías importantes que a continuación indicaremos. La primera de éstas es de localización. En la medida en que no sean homogéneas las condiciones ambientales naturales para el cultivo de productos agrícolas en una zona y en la medida en que la producción existente no se encuentre ordenada en términos de estas condiciones ambientales, habrá ventaja de costo resultante del aumento de la flexibilidad de la localización agrícola. Esta flexibilidad servirá también para reducir el costo social de la protección regional en comparación con la nacional. Esto se daría a través de los probables cambios en la calidad de la tierra disponible para la producción de diversas cosechas y también por la cantidad de tierra igualmente disponible. En estas situaciones la expansión tendría lugar a un costo social más bajo en la medida en que a) existan limitaciones importantes en cuanto a tierras utilizables en los territorios participantes considerados en forma separada, y $b$ ) al existir disponibilidad de este tipo de tierras, su costo de oportunidad sea más alto en un territorio en comparación con otro.

La segunda de estas economías de escala se obtiene a través de variaciones en el volumen del gasto de capital. En el caso de la industria, las economías de escala se han concentrado en la disminución de los costos de producción por unidad consecuentes con un incremento del tamaño del mercado. Es menos probable que se dé una fuente de economías de este tipo en la agricultura puesto que la tecnología generalmente usada no permite una correlación elevada entre ganancias de productividad en la producción y el tamaño del mercado. En el caso de la agricultura, la reducción del costo de capital por unidad necesario para iniciar la producción es la fuente de beneficios. Por lo tanto, para producir más a través de la expansión de la superficie bajo cultivo se necesitaría llevar a cabo fuertes gastos de capital en la preparación de la tierra, el suministro de agua, los caminos de acceso, 
la transportación y las construcciones rurales. El costo unitario de estos gastos de capital variará en relación con la extensión de tierra que se incorpore a la producción. Es poco probable que los costos totales varíen en proporción directa a la tierra incorporada a la producción, de manera que el costo de la preparación de 4000 Has. de tierra no utilizada no sería igual al doble del costo de preparar 2000 Has. Estas economías serán más grandes cuanto mayor sea la escala de operaciones permitida por el mercado regional en comparación con los mercados nacionales.

Una tercera fuente de economías de escala consiste en evitar la duplicación regional. Bajo una política de sustitución de importaciones nacional, la duplicación regional es inevitable. Bajo una política de sustitución de importaciones regional sólo es inevitable la duplicación internacional. Para la economía como un todo, la cuarta fuente de reducciones en los coeficientes de insumo surge de las ligas interindustriales entre la agricultura y el resto de la economía. Estas economías externas pueden ser generadas tanto por el lado de la demanda, por ejemplo, cuando los productos disfrutan de demanda complementaria, como por el lado de la oferta. En cuanto a demanda, la mayoría de las importaciones de las economías estructuralmente dependientes está constituida por alimentos de elevado contenido proteínico. La sustitución de importaciones, en tanto por otras razones Ileve a un costo real de producción más bajo en comparación con la sustitución de importaciones nacional, aumentará las economías que se obtendrán del consumo complementario de otros bienes producidos internamente, tales como condimentos, cultivos de tubérculos, etc. Desde el ángulo de la oferta existen dos fuentes posibles de ganancia. En primer lugar, podría haber una disminución generalizada en el costo unitario de un complejo de actividades. Esto se debería a la disminución de los coeficientes de insumo en aquella parte del grupo de actividades en que las economías de escala de la producción sean potencialmente importantes. Por lo tanto, podrían obtenerse economías de escala en el sector de las industrias alimenticias y en aquellos otros sectores de productos manufacturados que dependen de la producción agrícola, puesto que como actividades manufactureras, aquellas economías que se hacen posibles por una mayor especialización, etc., se ampliarán con el tamaño del mercado. En segundo lugar, puede haber una disminución del costo de los insumos de la agricultura. Esto puede suceder donde los insumos importantes de la agricultura sean productos importantes de la industria manufacturera; tres buenos ejemplos de esto son los fertilizantes, los alimentos para animales y el suministro de agua. Estas economías variarán directamente con las relaciones intersectoriales de la agricultura y con el tamaño del mercado. Con respecto a estas dos variables, la sustitución de importaciones regional es superior a la sustitución de importaciones nacional.

La quinta fuente de ganancias y la que potencialmente es más importante se deriva de las economías en la investigación y en la innovación científicas. En la literatura del crecimiento económico esta fuente de ganancias ha sido subestimada frecuentemente en relación con las ganancias de la acumulación. Sin embargo, aun un examen 
superficial de la situación de la agricultura en las economías pequeñas estructuralmente dependientes (incluyendo las cosechas de exportación), muestra una dependencia fuerte, casi proporcional, del aumento de la producción con respecto a los incrementos en la superficie de la tierra. Un ejemplo notable de esto es el cultivo del arroz en Guyana, donde las retribuciones al trabajo y a la tierra no se han alterado desde que se inició la industria en el siglo XIx. Se deduce de esto que no puede subestimarse el alcance de las economías en la investigación y en los estudios científicos en una agrupación regional.

La concentración de la capacidad de investigación tendrá importancia para la región desde dos aspectos principales. En primer lugar, si las capacidades técnicas y de investigación existentes no tienen la calidad necesaria se originarán dos tipos de desperdicio. En primera instancia, en un área de escasez considerable se da una duplicación en el sentido de que cada territorio trata de mantener su propio aparato de investigación. En segundo lugar, los recursos existentes tienden a estar demasiado dispersos en cada uno de los territorios. El resultado es que el desarrollo de las técnicas agrícolas ha sido demasiado lento y que hasta ahora no se han realizado avances satisfactorios en áreas muy importantes de sustitución de importaciones, por ejemplo, en la selección de especies de ganado lechero tropical. Se deduce de lo anterior que las economías de escala derivadas de la investigación pueden ser muy importantes.

La centralización de los esfuerzos de investigación regionales en la agricultura, que llevaría a una nueva orientación de la innovación científica, constituye la segunda razón por la que se podrían esperar beneficios significativos. El desarrollo de una tradición científica nacional en las economías pequeñas estructuralmente dependientes ha sido de limitada importancia en cuanto a la creación de un bienestar material para la gente. Donde se ha realizado investigación científica, ésta se ha dirigido hacia la investigación de las materias primas tradicionales de exportación. Así ha ocurrido aun cuando han existido, para satisfacer las demandas regionales, instituciones "locales" mundialmente famosas tales como el Colegio Imperial de Agricultura Tropical de Trinidad. La integración de la región con la integración concomitante de las facilidades de investigación necesitará una nueva orientación en la investigación científica a favor de productos con mayor potencial a largo plazo para estas áreas.

\section{V}

En conclusión, podemos señalar que las cuestiones teóricas planteadas en este trabajo implican modificaciones bastante radicales a la teoría de la integración existente así como a la del comercio internacional. Si la integración se efectuara en la forma así señalada, surgirán también algunas consecuencias prácticas bastante radicales, puesto que amenazaría las bases de las relaciones de comercio entre las economías económicamente "maduras" y las "inmaduras". De particular significado es que, al contrario de la práctica tradicional, esta reorganización de las relaciones de comercio no presupondría concesiones de mercado de parte de las economías económicamente "ma- 
duras". El énfasis en la planeación y la coordinación, y en factores tanto sociales como económicos, amenazaría también la imagen que la mayoría de los gobiernos de las economías estructuralmente dependientes tienen de sí mismos con respecto a su papel en el proceso económico. También en este sentido la solución al problema se sitúa dentro de posiciones bastante radicales. 